\title{
Catastrophic Risk Management: Flood and Seismic Risks Case Studies
}

Tatiana Ermolieva and Yuri Ermoliev<smiles>[R6][SbH]=[Os]</smiles>

October 2005

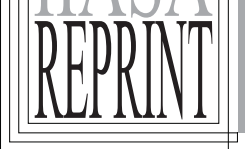





\section{Catastrophic Risk Management: Flood and Seismic Risks Case Studies}

Tatiana Ermolieva

Yuri Ermoliev

RP-05-007

October 2005

Reprinted from Applications of Stochastic Programming, Stein W. Wallace and William T. Ziemba, Editors, MPS-SIAM Book Series on Optimization 5.

International Institute for Applied Systems Analysis - Schlossplatz 1 - A-2361 Laxenburg • Austria Tel: (+43 2236) 807 • Fax: (+43 2236) 71313•E-mail: publications@iiasa.ac.at•Web: www.iiasa.ac.at 
IIASA Reprints make research conducted at the International Institute for Applied Systems Analysis more accessible to a wider audience. They reprint independently reviewed articles that have been previously published in journals. Views or opinions expressed herein do not necessarily represent those of the Institute, its National Member Organizations, or other organizations supporting the work.

Reprinted with permission from Applications of Stochastic Programming, Stein W. Wallace and William T. Ziemba, Editors, MPS-SIAM Book Series on Optimization 5.

Copyright $\odot 2005$ Society for Industrial and Applied Mathematics.

All rights reserved. No part of this publication may be reproduced or transmitted in any form or by any means, electronic or mechanical, including photocopy, recording, or any information storage or retrieval system, without permission in writing from the copyright holder. 


\title{
Chapter 22
}

\section{Catastrophic Risk Management: Flood and Seismic Risks Case Studies}

\author{
Tatiana Ermolieva* and Yuri Ermoliev*
}

\subsection{Introduction}

Losses from human-made and natural catastrophes are rapidly increasing. Within the last three decades the direct damages only from natural disasters have increased ninefold (see [25, 34]). The main reason for this is the clustering of people and capital in hazard-prone areas as well as the creation of new hazard-prone areas, a phenomenon that may be aggravated by a lack of knowledge of the risks. It is estimated (see [35]) that within the next 50 years more than a third of the world population will live in seismically and volcanically active zones. Analysis of insurance companies shows that because of economic growth in hazard-prone areas, damages due to natural catastrophes have grown at an average annual rate of $5 \%$ [19]. The possibility of more frequent catastrophes dominates discussions of current global changes. In fact, the main point of the climate change debates concerns the increasing frequency of extreme floods, droughts, and windstorms rather than the increasing global mean temperature which can be within the difference between the average temperature of cities and their surrounding rural areas. Another source of catastrophes is associated with increasing interdependencies among different countries. Dantzig [9] compares our society with a busy highway where disruptions in one of its parts may lead to fundamental traffic jams in other parts.

The increasing vulnerability of our society calls for new integrated approaches to economic developments and risk management with an explicit emphasis on the possibility of catastrophes. The standard economic theory is dominated by truncated models of uncertainties, represented by a finite manageable number of contingencies well known to the whole society, which can, therefore, be priced and spread over the whole society through

\footnotetext{
*International Institute for Applied Systems Analysis (IIASA), A-2361 Laxenburg, Austria (ermol@iiasa.ac.at, ermoliev@iiasa.ac.at).
} 
markets. Under such assumptions of certainty, catastrophes pose no special problems [3].

Insurance risk theory has developed independently of the fundamental economic ideas (see discussion in $[3,20]$ ). The central problem of this theory is modeling the probability distribution of total future claims [23], which is then used to evaluate ruin probabilities, premiums, reinsurance arrangements, etc. This theory essentially relies on the assumption of independent, frequent, low-consequence (conventional) risks, such as car accidents, for which decisions on premiums, estimates of claims, and likelihood of insolvency (probability of ruin) can be calculated by using rich historical data. The frequent conventional risks also permit simple "more-risks-are-better" strategies with simple trial-and-error or learning-bydoing procedures for adjusting insurance decisions.

Catastrophes produce losses highly mutually dependent in space and time, which challenges the standard risk pooling concepts and the standard extremal value theory [11]. The law of large numbers does not operate (in general), and the probability of ruin can be reduced not just by pooling risks but only if insurers deliberately select the dependent fractions of catastrophic risks they will cover. The existing extremal value theory deals also primarily with independent events assuming these events are quantifiable by a single number. Definitely catastrophes are not quantifiable events in this sense. They may have quite different spatial and temporal patterns, which cause significant heterogeneity of losses in space and time. These losses can be dramatically affected by risk mitigation decisions (say, by construction of a dike or a flood retention area) and loss spreading schemes within a country or on the international level through the insurance or financial markets.

Catastrophe modeling [43] is becoming increasingly important in risk management for estimating dependent catastrophic losses and making decisions on the allocation of coverage, premiums, reinsurance agreements, and the effects of mitigation measures.

The aim of this paper is to show that the choice of decisions in the presence of catastrophic risks can be regarded as a stochastic optimization problem. This discussion closely follows the papers $[1,2,14,15,17,18]$. Section 22.2 illustrates the peculiarities of emerging stochastic optimization problems by using a typical model of risk theory. Section 22.3 provides more motivations by outlining important case studies. Sections 22.4 and 22.5 discuss a rather general model attempting to bridge decision-oriented economic theory with risk theory and catastrophe modeling. Risk management decisions are evaluated from perspectives of the welfare growth in the region. We use such economically sound risk measures as expected costs of overpayments and borrowing, which have strong connection with standards in the insurance business, the insolvency and stability constraints, and the conditional-value-at-risk (CVaR) type of risk measures. Section 22.6 discusses the results of the case study in the seismic-prone Toscana region of Italy. Section 22.7 outlines the computational procedure.

\subsection{The standard insurance risk model}

Consider a simple model of growth under shocks, which is a stylized version of insurance business [10]. The main variable of concern is the risk reserve $r^{t}$ at time $t: r^{t}=r_{0}+\pi^{t}-A^{t}$, $t \geq 0$, where $\pi^{t}, A^{t}$ are aggregated premiums and claims, and $r_{0}$ is the initial risk reserve. The process $A^{t}=\sum_{k=1}^{N(t)} S_{k}$, where $N(t), t \geq 0$, is a counting process for a number of claims in interval $[0, t]$ (e.g., a Poisson process) with $N(0)=0$, and $\left\{S_{k}\right\}_{1}^{\infty}$ is a sequence of 
independent and identically distributed random variables (claims), in other words, replicates of a random variable $S$. The inflow of premiums $\pi^{t}$ pushes $r^{t}$ up, whereas the random outflow $A^{t}$ pushes $r^{t}$ down.

The main problem of risk theory $[10,23]$ is the evaluation of the ruin probability $\Psi=$ $P\left\{r^{t} \leq 0\right.$ for some $\left.t, t>0\right\}$ under different assumptions on $\pi^{t}, A^{t}$. There are several cases where $\Psi$ can be explicitly given, or at least given in a form suited for numerical calculations. An important case arises when the claim distribution is a mixture of exponential distributions and claims occur according to a Poisson process. There are numerous approximations for the probability distribution of $A^{t}$. Most of them provide satisfactory results only in the area of mean values and cannot be applied to catastrophes.

The typical actuarial analysis is based on the following. Assume that $N(t)$ and $S_{k}$ are independent, $N(t)$ has intensity $\alpha$, i.e., $E\{N(t)\}=\alpha t$, and $\pi^{t}=\pi t, \pi>0$. Then the expected profit over the interval $[0, t]$ is $(\pi-\alpha E S) t$; that is, the expected profit increases in time for $\pi-\alpha E S>0$. The difference $\pi-\alpha E S$ is the "safety loading." The strong law of large numbers implies that $\left[\pi^{t}-A^{t}\right] / t \rightarrow[\pi-\alpha E S]$ with probability 1 . Therefore, in the case of positive safety loading, $\pi>\alpha E S$, we have to expect that the real random profit $\pi^{t}-$ $A^{t}$ for large enough $t$ would also be positive under the appropriate choice of premium $\pi=$ $(1+\rho) \alpha E S$, where $\rho$ is the "relative safety" loading, $\rho=(\pi-\alpha E S) / \alpha E S$. But this holds only if the ruin does not occur before time $t$. This is a basic actuarial principle: premiums are calculated by relying on the mean value of aggregated claims increased by the (relative) safety loading. Thus, practical actuarial approaches ignore complex interdependencies among timing of claims, their sizes, and the possibility of ruin, $r^{t} \leq 0$. The random jumping process $r^{t}$ is simply replaced by a linear in $t$ function $\bar{r}^{t}=r_{0}+(\pi-\alpha E S) t$.

Various decision variables affect $\Psi$. Claim size $S$ depends on the coverages of the insurer from different locations. Important decision variables are $r_{0}, \pi$, and reinsurance arrangements, for example, the "excess of loss" reinsurance contract. In this case, the insurer retains only a portion, $S(x)=\min \{S, x\}, x \geq 0$, of a claim $S$, and the remaining portion is passed to the reinsurer. The reinsurance contracts with deductibles are defined by two variables $x=\left(x_{1}, x_{2}\right)$. In this case $S(x)=\max \left\{x_{1}, \min \left[S, x_{2}\right]\right\}-x_{1}, x_{1} \geq 0$, $x_{2} \geq 0$ is retained by the insurer. The reduction of $\Psi$ to acceptable levels can be viewed as a chance constraint problem [38]. The complexity is associated with the jumping process $A^{t}$ with analytically intractable dependencies of $A^{t}$ on decision variables, which restricts the straightforward use of conventional stochastic optimization (STO) methods. The direct sample mean estimation of $\Psi(x)$ requires a very large number of observations and leads to discontinuous functions. The following simple idea can be used for rather general problems to overcome these difficulties.

Consider $t=0,1, \ldots$, and assume that $r^{t}$ can be subdivided into a "normal" part (including $r_{0}$ ), $M^{t}$, associated with ordinary claims, and a "catastrophic" part $B^{t}, \pi^{t}=\pi t$, where $\pi$ is the rate of premiums related to catastrophes; the probability of a catastrophic event $p$ is characterized by a probability distribution in an interval $[\underline{p}, \bar{p}]$, and the probability distribution $V_{t}(z)=P\left[M^{t}<z\right]$ can be evaluated. Assume also that ruin may occur only due to a catastrophe. Then the probability of ruin after the first catastrophe and with the excess of loss contract is defined as a function

$$
\Psi(x)=E \sum_{t=1}^{\infty} p(1-p)^{t-1} V_{t}\left(\min \left\{x, B^{t}\right\}-\pi t\right) .
$$


In general cases we can use an arbitrary "small” auxiliary random variable instead of $M^{t}[14,15]$. Definitely, $(22.1)$ enables us to evaluate $\Psi(x)$ much faster (fast Monte Carlo sampling) in contrast to direct evaluation of $\Psi(x)$ by straightforward Monte Carlo sampling. This is the main idea in dealing with rare catastrophic events. The search for a desirable $x$ can be based on methods outlined in section 22.7.

\subsection{Overview of case studies}

To better understand the main features of the model described in the next section, let us sketch out some case studies [1, 2, 17] carried out at the International Institute for Applied Systems Analysis (IIASA). The main concern of these case studies is related to issues emphasized by Froot in [19]. Froot admitted that most of the catastrophic losses

are paid ex-post by some combination of insurers and reinsurers (and their investors), insured, state and federal agencies and taxpayers, with only some of these payments being explicitly arranged ex-ante. This introduces considerable uncertainty about burden sharing into the system, with no particular presumption that the outcome will be fair. The result is incentives for players to shift burdens towards others, from the homeowner who builds on exposed coastline, to insurers who write risks that appear highly profitable in the absence of a large event. ... But most importantly, bad or inefficient risk sharing raises the cost of capital for companies and requires returns for households, reducing the amount of profitable investments and the rate of growth of the economy... it is worth noting that the gains from higher growth rate are huge...

For Hungary [24], facing special problems of a poor and immobile population, ex ante mechanisms to fund the costs of recovery and, in particular, the establishment of a multipillar flood loss-sharing program, are especially important. In the analysis of the Upper Tisza river pilot region [17] it is assumed, in particular, that for the first pillar the government would provide compensation of a limited amount to all households that suffer losses from flooding. As the second pillar, a special regional fund would be established through a mandatory public flood insurance on the basis of location-specific risk exposures. It is assumed that the governmental financial aid is regulated through this fund. As a third pillar, a contingent credit may also be available to provide an additional injection of capital to stabilize the system. In the latter case, the lender charges a fee that the borrower (in our case, the fund) pays as long as the trigger event does not occur. If the event does occur, the borrower rapidly receives the fund. The advantages of this financial arrangement in contrast to catastrophic bonds are discussed, e.g., in [37].

Such a program would increase the responsibility of individuals and local governments for flood risks and losses. Local governments may be more effective in the evaluation and enforcement of loss-reduction and loss-spreading measures, but this is possible only through location-specific analysis of potential losses, the mutual interdependencies of these losses, and the sensitivities of the losses to new risk management strategies.

The lack of historical data is a main challenge in dealing with rare catastrophes. Purely adaptive, learning-by-doing types of approaches may be very expensive and dangerous. The development of models can be viewed as a mitigation measure enabling the simulation of probable catastrophes for designing ex ante preparedness programs. 
The analysis of possible gains and losses from different arrangements of the program outlined above is a multidisciplinary task, which has to take into account the frequency and intensity of hazards, the stock of capital at risk, its structural characteristics, and different measures (in particular, engineering, financial) of vulnerability. These efforts require the development of so-called catastrophe models [43]. For this purpose, Ermolieva et al. [17] discuss a GIS-based catastrophe model developed for the Upper Tisza pilot region that, in the absence of historical data, simulates samples of mutually dependent potential losses at different locations. The model emphasizes the cooperation of various agents in dealing with catastrophes. The solution to catastrophic risk management, especially for small economies with limited risk absorption capacity, cannot be accomplished (see $[2,8,37]$ ) without pooling of risk exposures. The proposed model involves pooling risks through mandatory flood insurance based on location-specific exposures, partial compensation to the flood victims by the central government, and a contingent credit to the pool. This program encourages accumulation of regional capital to better buffer international reinsurance market volatility. To stabilize the program such economically sound risk indicators as expected overpayments by individuals (cells of flood-prone areas) and an expected shortfall of the mandatory insurance are used. These indicators together with the so-called stopping times orient the analysis toward the most destructive scenarios. It was shown (see $[14,15]$ and references therein) that the explicit introduction of ex post borrowing (see also section 22.5) as a measure against insolvency enables us to approximate the insolvency constraint by a convex optimization problem, whereas the use of the contingent credit leads to the CVaR type of risk measures.

For the seismic-prone Irkutsk region in Russia [2], the focus of the analysis was on the feasibility of an insurance pool to cover catastrophic losses subject to strong standard insolvency regulations. In contrast to the case study in Hungary, the contribution of different insurers to the stability of the pool was explicitly analyzed by taking into account the transaction costs and effects of mutual dependencies among claims from different locations (cells).

Many authors have stressed the need for better models to improve established insurance practices for evaluating catastrophe coverages (see, for instance, [43]). Such models can be even more critical for guidance and setting regulations in countries that are moving toward market economies. In Russia [2] new legislative instruments and government resolutions are creating a framework for risk management similar to that existing in the OECD countries. However, in Russia and other transition countries the emergence of a viable insurance industry is slow and subject to insolvency risks due to problems of the national economies, the lack of consolidated experience and practicable guidance, and the lack of sufficient risk reserves of the existing companies. For example, when insurance is available in seismic regions, premiums neither are based on the probability of occurrence of earthquakes nor do they differentiate among geological situations and construction type. The model proposed in $[1,2]$ is a pilot exercise, which, however, can create the basis for cooperation among researches, insurers, and regulatory bodies in transition countries. In the case of Russia's emerging insurance industry, cooperation among insurers will undoubtedly play an important role in stabilizing the insurance market. A key problem, however, is the lack of necessary information on the distribution of losses among locations. For this purpose the region-specific earthquake generator (see $[5,42]$ ) was designed and incorporated within the STO model $[1,2]$. 
The case study for the seismic-prone Italian region especially illustrates the fact that neither the market nor the government will be acceptable as the mechanism for catastrophic risk management. Thus, some form of a public-private partnership may be appropriate [30].

A well-known example of a government acting as a primary insurer is the United States National Flood Insurance Program (NFIP), which seeks to provide insurance at actuarially fair premiums combined with incentives for communities and homeowners to take appropriate loss-reducing measures. Given the size of the United States and the large number of persons living in flood plains, the program is sufficiently diversified to cover most regional losses with premium payments. In contrast to the NFIP, some government insurance schemes in Europe, e.g., the French national insurance program, cross subsidize claims. This is because the French constitution $(1946,1958)$ established the principle of "the solidarity and equality of all French citizens facing the expenses incurred through national calamities" [21].

However, even if many governments are pursuing policies to reduce their role in compensating victims, a study [31] confirms that the victims and their governments bear the major losses from natural disasters and, worldwide, there is only moderate risk transfer with insurance. An important consideration for national insurance strategies is linking private insurance with mitigation measures to reduce losses. Insurers, however, are reluctant to enter markets that expose them to a risk of bankruptcy. In the United States, for example, many insurers have pulled out of catastrophic risk markets in response to their large losses from natural catastrophes in the last decade [25].

To reduce their risk of insolvency, insurers' strategies may be based on modeling tools that account for the complexity implied by the manifold dependencies in the stochastic process of catastrophic events, decisions, and losses. For example, to study the problem in its complexity for the Toscana region, a spatial-dynamic, stochastic optimization model has been developed in $[13,14,16,18]$ and is described below.

In Italy, a law for integrating insurance in the overall risk management process was proposed only in late 1997 (within the Design of Law 2793: "Measures for the stabilization of the public finance"). This opened a debate, which has not yet been concluded by a legislative act. Therefore policy options for a national insurance strategy are still open to investigation. The Institute for Research on Seismic Risk of the Italian National Research Council made data from a previous study available [36]. These have been incorporated into a Monte Carlo catastrophe model, which simulates occurrence of earthquakes affecting the region, calculates attenuation according to the geological characteristics, and finally determines the acceleration at the ground in each municipality. The model explicitly incorporates the vulnerability of the built environment, with data on number and types of buildings in each municipality of the region.

Since the discussion of GIS-based catastrophe models deserves a separate paper, our analysis in the next sections is concentrated primarily on the evaluation of different catastrophic risk management policies assuming that mutually dependent spatial losses are generated in time and space (cells) by a catastrophe model.

\subsection{STO model}

Catastrophes may lead to large costs, social disruption, and economic stagnation. A catastrophe would ruin many agents if their risk exposures were not properly managed. To design 
safe catastrophic risk management strategies it is necessary to define at least the following: patterns of possible disasters in space and time, a map of regional values and their vulnerability, and feasible decisions, e.g., insurance coverages. The model of this section uses this information. It emphasizes the collective nature of catastrophe risk management. The aim of this model is to address only main features of the problem. Basically, we assume that the goal of the insurance sector is to maximize its wealth while maintaining survival and stability of growth (see also the discussion in [41]). In a similar manner, other agents are concerned with their sustained wealth growth, whereas the main concern of the government is the sustained welfare growth of the region. The model emphasizes catastrophe risk management as a long-term business rather than as subject of annual accounting and taxation. Accordingly, catastrophe reserves should be accumulated over years.

Assume that the study region is divided into subregions or cells $j=\overline{1, m}$. A cell may correspond to a collection of households, a zone with similar seismic activity, a watershed, a grid with a segment of a gas pipeline, etc. The choice of cells provides a desirable representation of losses. For each cell $j$ there exists an estimate of its wealth at time $t$ that may include the value of infrastructure, houses, factories, etc. A sequence of random catastrophic events $\omega=\left\{\omega_{t}, t=\overline{0, T-1}\right\}$ affects different cells $j=\overline{1, m}$ and generates at each $t=\overline{0, T-1}$ mutually dependent losses $L_{j}^{t}(\omega)$, i.e., damages of the wealth at $j ; T$ is a time horizon. These losses can be modified by various decision variables. Some of the decisions reduce losses, say, a dike, whereas others spread them on a regional, national, and international level, e.g., insurance contracts, catastrophe securities, credits, and financial aid. If $x$ is the vector of the decision variables, then the losses $L_{j}^{t}(\omega)$ are transformed into $L_{j}^{t}(x, \omega)$. For example, we can think of $L_{j}^{t}(x, \omega)$ as $L_{j}^{t}(\omega)$ being affected by the decisions of the insurance to cover losses from a layer $\left[x_{j 1}, x_{j 2}\right]$ at a cell $j$ in the case of a disaster at time $t$ :

$$
L_{j}^{t}(x, \omega)=L_{j}^{t}(\omega)-\max \left\{x_{j 1}, \min \left[x_{j 2}, L_{j}^{t}\right]\right\}+x_{j 1}+\pi_{j}^{t},
$$

where $\max \left\{x_{j 1}, \min \left[x_{j 2}, L_{j}^{t}\right]\right\}-x_{j 1}$ are retained by insurance losses, and $\pi_{j}^{t}$ is the premium. In the most general case, the vector $x$ comprises decision variables of different agents, including governmental decisions, such as the height of a new dike or a public compensation scheme defined by a fraction of total losses $\sum_{j=1}^{m} L_{j}^{t}$. The insurance decisions concern premiums paid by individuals and the payments of claims in the case of catastrophe. There are complex interdependencies among these decisions, which call for the cooperation of agents. For example, the partial compensation of catastrophe losses by the government enforces decisions on loss reductions by individuals and, hence, increases the insurability of risks and helps the insurance industry to avoid insolvency. On the other hand, the insurance combined with risk-reduction measures can reduce losses, compensations, and governmental debt and stabilize the economic growth of the region and the wealth of individuals.

We assume that $\omega$ is an element of a probability space $(\Omega, \mathcal{F}, P)$, where $\Omega$ is a set of all possible $\omega$ and $\mathcal{F}$ is a $\sigma$-algebra of measurable (with respect to probability measure $P$ ) events from $\Omega$. Let $\left\{\mathcal{F}_{t}\right\}$ be a nondecreasing family of $\sigma$ algebras, $\mathcal{F}_{t} \subseteq \mathcal{F}_{t+1}, \mathcal{F}_{t} \subseteq \mathcal{F}$. Random losses $L_{j}^{t}(\omega)$ are assumed to be $\mathcal{F}_{t}$-measurable; i.e., they depend on the observable catastrophes until time $t$. In the following we specify dependencies of these variables on $\omega$, although sometimes we do not use $\omega$ when these dependencies are clear from the text.

Catastrophe losses are shared by many participants, such as individuals (cells), governments, insurers, reinsurers, and investors. In the model we call them "agents," since the 
main balance equations of our model are similar for all of them. For each agent $i$ a variable of concern is the wealth $W_{i}^{t}$ at time $t=\overline{0, T}$

$$
W_{i}^{t+1}(\omega)=W_{i}^{t}(x, \omega)+I_{i}^{t}(x, \omega)-O_{i}^{t}(x, \omega), \quad i=\overline{1, n}, \quad t=\overline{0, T-1}, \quad \omega \in \Omega,
$$

where $W_{i}^{0}$ is the initial wealth. This is a rather general process of accumulation, which, depending on the interpretation, can describe the accumulation of reserve funds, the dynamics of contamination, or processes of economic growth with random disturbances (shocks), reserves of the insurance company at moment $t$, the gross national product of a country, or the accumulated wealth of a specific region. In more general cases, when catastrophes may have profound effects on economic growth, this model can be generalized to an appropriate version of economic-demographic model (see, for example, in [32]) enabling it to represent movements of individuals and the capital accumulation processes within the economy.

For the simplicity of the exposition we do not discuss discount rates in these equations since catastrophes require nonstandard approaches. In particular, induced by catastrophes, discount rates become important, which is evident from the evaluation (22.1). We use also the same index $i$ for quite different agents. Therefore, the variables $I_{i}^{t}(x, \omega), O_{i}^{t}(x, \omega)$ may have quite a different meaning. For example, for each insurer $i$ we can think of $I_{i}^{t}$ as premiums $\pi_{i}^{t}$ which are ex ante arranged and do not depend on $\omega$, whereas $O_{i}^{t}$ is defined by the claim size $S_{i}^{t}$ and possible transaction costs which triggers a random jump of the risk reserve $W_{i}^{t}$ (usually denoted as $R_{i}^{t}$ ) downward at random times of catastrophic events (as in the simple model of section 22.2). If $i$ corresponds to a cell, then income $I_{i}^{t}$ may be affected by a catastrophic event $\omega$ generated by a catastrophe model. The incomes $I_{i}^{t}$ can be defined by a set of scenarios or through a regional growth model with geographically explicit distribution of the capital among cells. The term $O_{i}^{t}$ may include losses $L_{i}^{t}$, taxes and premiums paid by $i$. For central or local governmental agent $i$ (e.g., mandatory insurance, catastrophe fund) $I_{i}^{t}$ may include a portion of taxes collected by the government (compensations of losses by the government), and $O_{i}^{t}$ may consist of mitigation costs, debts, loans, and fees paid for ex ante contingent credits.

Catastrophes may cause strong dependencies among claims $S_{i}^{t}$ for different insurers $i$. These claims are defined by decisions on coverages of losses $L_{j}^{t}$ from different locations $j$. For example, let us denote by $x_{i j}^{t}$ a searched fraction of $L_{j}^{t}$ covered by insurer $i$, e.g., assume $i=\overline{1, n}$. Then

$$
\sum_{i=1}^{n} x_{i j}^{t} \leq 1, x_{i j}^{t} \geq 0, \quad j=\overline{1, m},
$$

and claims $S_{i}^{t}$ are linear functions of $x=\left\{x_{i j}^{t}, i=\overline{1, n}, j=\overline{1, m}, t=\overline{0, T-1}\right\}$ :

$$
S_{i}^{t}(x, \omega)=\sum_{j=1}^{m} L_{j}^{t} x_{i j}^{t}, \quad i=\overline{1, n}, \quad t=\overline{0, T-1} .
$$

If $I_{i}^{t}, O_{i}^{t}$ simply correspond to premiums $\pi_{i}^{t}$ and claims $S_{i}^{t}$, then the wealth of insurer $i$ (its risk reserves) are calculated for $t=\overline{0, T-1}, \omega \in \Omega$ as follows:

$$
R_{i}^{t+1}(x, \omega)=R_{i}^{t}(x, \omega)+\sum_{j=1}^{m} \pi_{i j}^{t} x_{i j}^{t}-\sum_{j=1}^{m} L_{j}^{t}(\omega) x_{i j}^{t},
$$


where $\pi_{i j}^{t}$ are rates of premiums per unit of coverage.

For each $i$ consider a stopping time $\tau_{i}$ for process $W_{i}^{t}(x, \omega)$, i.e., a random variable with integer values, $t=\overline{0, T}$. The event $\left\{\omega: \tau_{i}=t\right\}$ with fixed $t$ depends only on the history till $t$, and it corresponds to the decision to stop process $W_{i}^{t}(x, \omega)$ after time $t$. Therefore, $\tau_{i}$ in the case of $W_{i}^{t}$ defined according to (22.4) depends on $\left\{x_{i j}^{k}, i=\overline{1, n}, j=\overline{1, m}, k=\overline{0, t}\right\}$; i.e., it is a function $\tau_{i}(x, \omega)$. Examples of $\tau_{i}$ may be $\tau_{i}=T$, the time of the first catastrophe, or the time of the ruin before a given time $T: \tau_{i}(x, \omega)=\min \left\{T, \min \left[t: W_{i}^{t}(x, \omega)<0\right.\right.$, $t>0]\}$. The last example defines $\tau_{i}$ as a rather complex implicit function of $x$.

Assume that each agent $i$ maximizes (possibly negative) wealth at $t=\tau_{i}$. The notion of wealth at $t$ requires exact definition since it must represent, in a sense, the whole probability distribution $W_{i}^{t}$. The traditional expected value $E W_{i}^{t}$ may not be appropriate for probability distributions of $W_{i}^{t}$ affected by rare catastrophes of high consequences. As a result they may have a multimode structure with "heavy tails." We can think of the estimate for $W_{i}^{t}$ as a maximal value $V_{i}^{t}$, which does not overestimate, in a sense, random value $W_{i}^{t}$, i.e., cases when $\min _{s \leq t}\left(W_{i}^{t}(q, \omega)-V_{i}^{t}\right)<0$. Formally, $V_{i}^{t}$ can be chosen by maximizing

$$
V+\gamma E \min \left\{0, W_{i}^{t}-V\right\}
$$

or a more general function $V+\gamma E d\left(W_{i}^{t}-V\right)$ for appropriate function $d(\cdot)$ and $\gamma>0$. The second term can be considered as the risk of overestimating the wealth $W_{i}^{s}(x, \omega)$ for $s=0,1, \ldots, t$. This concept corresponds to the CVaR risk measure (see $[4,27,40])$. The maximization of (22.5) is a simple example of the so-called stochastic maximin problems. It is easy to see from the optimality conditions for this problem (see [12, pp. 165, 416]) that for continious distributions the optimal value $V$ satisfies condition $P\left[W_{i}^{t} \leq V\right]=1 / \gamma$. For the normal distribution and $\gamma=2$, it coincides with the traditional mean value $E W_{i}^{t}$. In the case of quadratic function $d(\cdot)$ and $\gamma=\infty$, i.e., the maximization of $E\left(W_{i}^{t}-V\right)^{2}$, the optimal $V=E W_{i}^{t}$.

Besides the maximization of wealth, the agent $i$ is concerned with the risk of insolvency, i.e., when $W_{i}^{s}<0$ for some $s=0,1, \ldots, t$, as well as the lack of sustained growth, i.e., when $I_{i}^{s}-O_{i}^{s}<0$ for some $s=0,1, \ldots, t$. In accordance with this consider the stochastic goal functions

$$
\begin{aligned}
f_{i}^{t}(x, V, \omega)= & V_{i}^{t}+\gamma_{i} \min \left\{0, \min _{s \leq t}\left[W_{i}^{s}(x, \omega)-V_{i}^{s}\right]\right\}+\delta_{i} \min \left\{0, \min _{s \leq t} W_{i}^{s}(x, \omega)\right\} \\
& +\beta_{i} \min \left\{0, \min _{s \leq t}\left[I_{i}^{s}(x, \omega)-O_{i}^{s}(x, \omega)\right]\right\} \\
F_{i}(x, V)= & E f_{i}^{\tau_{i}(x, \omega)}(x, V, \omega)
\end{aligned}
$$

where nonnegative $\gamma_{i}, \delta_{i}, \beta_{i}$ are substitution coefficients between wealth $V_{i}^{t}$ and risks of overestimating wealth, insolvency, and overestimating sustained growth. If a catastrophe is considered as the most distractive event, then we can use in the definition of $f_{i}^{t}$ simply $s=t$ instead of $\min _{s \leq t}$. These requirements reflect survival and stability constraints of agents. In (22.6) we use a modified form of (22.5), which is more appropriate for dynamic problems. Each agent attempts to maximize $F_{i}(x, V)$.

Pareto optimal improvements of risk situations with respect to goal functions $F_{i}(x, V)$ 
of different agents can be achieved by maximizing

$$
W(x, V)=\sum_{i=1}^{n} \alpha_{i} F_{i}(x, V)
$$

for different weights $\alpha_{i} \geq 0, \sum_{i=1}^{n} \alpha_{i}=1$. These weights reflect the importance of the agents. The maximization of $W(x, V)$ for different weights $\alpha_{i}, i=\overline{1, n}$, corresponds to a stochastic version of the welfare analysis [22].

When $n>1$, this model generalizes Borch's [7] fundamental ideas of risk sharing to the case of catastrophic risks. In the Borch model risks from different locations are substitutable, and the insurance pool is concerned only with the redistribution of the total risk mass. According to (22.3), our model emphasizes differences among risks from different locations, i.e., $m>1$ in contrast to $m=1$ of Borch's model.

Random functions $f_{i}^{t}(x, V, \omega)$ have a complex nested analytically intractable structure defined by simulated patterns of catastrophes. Their nonsmooth character is due to the presence of operators min and stopping times $\tau_{i}$, which may be complex implicit functions of $(x, \omega)$. When $f_{i}^{t}(x, V, \omega)$ are concave functions in $x$ as min of linear functions, then expectations $F_{i}(x, V)=E f_{i}^{t}(x, V, \omega)$ are also concave functions in $x$ for fixed $t$. The use of stopping times, $t=\tau_{i}$, generally destroys their concavity and even continuity. If stopping times do not depend on $x$, then these expectations are also concave. The use of such risk functions as in (22.5) is similar to the [33] mean-semivariance model and the [29] model with absolute deviations. Connections of problems (22.5) with the CVaR risk measure are established in [40].

The choice of weights (risk coefficients) $\gamma_{i}, \delta_{i}, \beta_{i}$, provides different trade-offs between wealth and risks. The increase of these parameters better eliminates corresponding risks.

\subsection{Insolvency, stopping time, and nonsmooth risk functions}

A key issue for selecting catastrophic risk portfolios is the financial ruin of insurers. It was shown (see [13]), that when risk coefficients $\gamma_{i}, \delta_{i}, \beta_{i}$ in (22.7) become large enough, then the probability of associated risks, in particular the probability of ruin, drops below a given level $p$ :

$$
P\left[\min _{s \leq \tau_{i}} W_{i}^{s}<0, i=\overline{1, n}\right] \leq p .
$$

The maximization problem defined by (22.6)-(22.7) is much simpler than the problem defined in terms of the chance constraint (22.8). The functions $F_{i}(x, V)$ defined according to (22.6) for $W_{i}^{t}(x, \omega):=R_{i}^{t}(x, \omega)$ are concave, whereas constraints (22.8) for the same case may have discontinuitous character, e.g., if $\omega$ has a discrete distribution. The problem defined in terms of the chance constraints (22.8) has a convex feasible set only under a strong assumption on the probability measure.

The discontinuous nature of the problem (22.6)-(22.7) may still be connected with the stopping time defined as the ruin (insolvency) moment. Different smoothing techniques 
for this case are analyzed in [15]. In particular, a rather natural idea of smoothing consists of introducing the possibility of borrowing money in the case of insolvency. It is natural to expect that when the payment for borrowing is high, agents will tend to exclude such a necessity through a reasonable selection of the portfolios, i.e., to keep constraints on the insolvency within reasonable limits. Let us slightly modify the process (22.2):

$$
W^{t+1}(x, y, \omega)=W^{t}(x, y, \omega)+I^{t}(x, \omega)-O^{t}(x, \omega)+y_{t+1}-\left(1+\beta_{t}\right) y_{t},
$$

where for the simplicity of notation we do not use here index $i, y_{t}$ is a value of borrowing on the interval $[t-1, t), \beta_{t}$ is the bank interest for the credit on the interval $[t-1, t)$, and $y=\left\{y_{0}, \ldots, y_{T}\right\}$. According to (22.9), the borrowing taken out at the moment $t$ to maintain solvency should be paid off at the next instant of time $t+1$ with interest $\beta_{t}$. If the reserves of the company are not sufficient for this purpose, then new loans are taken. The following fact is the key for dealing with discontinuities of the stopping time effects and the insolvency constraints. Let us represent the process $W^{t}(x, y, \omega)$ as

$W^{t}(x, y, \omega)=\widetilde{W}^{t}(x, \omega)-\sum_{s=1}^{t-1} \beta_{s} y_{s}+y_{t}, \quad \widetilde{W}^{t}(x, \omega)=W^{0}+\sum_{s=1}^{t}\left(I^{s}(x, \omega)-O^{s}(x, \omega)\right)$

and let $\left(x^{*}(\beta), V^{*}(\beta), y^{*}(\beta)\right)$ be a solution of the following problem: maximize

$$
F(x, V)=E \max _{y \geq 0}\left[f^{T}(x, V, y, \omega)-\left(1+\beta_{T}\right) y_{T}\right], \quad W^{t}(x, y, \omega) \geq 0, \quad 0 \leq t \leq T,
$$

where $f^{t}(x, V, y, \omega)$ is defined as in (22.6) for $W^{t}(x, y, \omega)$ defined according to (22.9).

Theorem 22.1 (see [15]). Assume that $\bar{R}_{t}(0) \geq 0, P\left[\widetilde{W}^{t}(x, \omega)=0\right]=0$, for any $x \in X$, $t=\overline{1, T}$. Then the probability of borrowing can be arbitrarily small by taking interest coefficients $\beta_{t}, t=\overline{1, T}$, large enough, i.e., $P\left[\widetilde{W}^{t}\left(x^{*}\left(\beta_{t}\right), \omega\right) \geq 0, t=\overline{1, T}\right] \rightarrow 1$ almost surely for $\min _{1 \leq t \leq T} \beta_{t} \rightarrow \infty$.

\subsection{The Tuscany region case study}

We now specify the general model described in section 22.4 to the Tuscany region. The region has been subdivided into $M \approx 300$ subregions, which correspond to the number of its municipalities. For each municipality $j$, number and types of buildings, their vulnerability, and number of built cubic meters are available. These represent the estimate of wealth $W_{j}$ in the municipality $j$. Using data and models in [36], a catastrophe generator has been created (see $[2,5,42]$ ) using the Gütenberg-Richter law and the attenuation characteristics of the region (see Figure 22.1). This enables us to generate the occurrences of earthquakes at random time moments, intensities, and accelerations in each municipality. The generator could be easily adapted to incorporate different kinds of distributions, non-Poissonian catastrophic processes, as well as microzoning within a municipality. It produces earthquake scenarios at random time moments according to geophysical characteristics of faults and soil type.

Simulated in time and space, earthquakes $\omega_{0}, \ldots, \omega_{t}$ may occur in different municipalities, inside or outside the region, have random magnitudes, and, therefore, affect a random number of municipalities. 


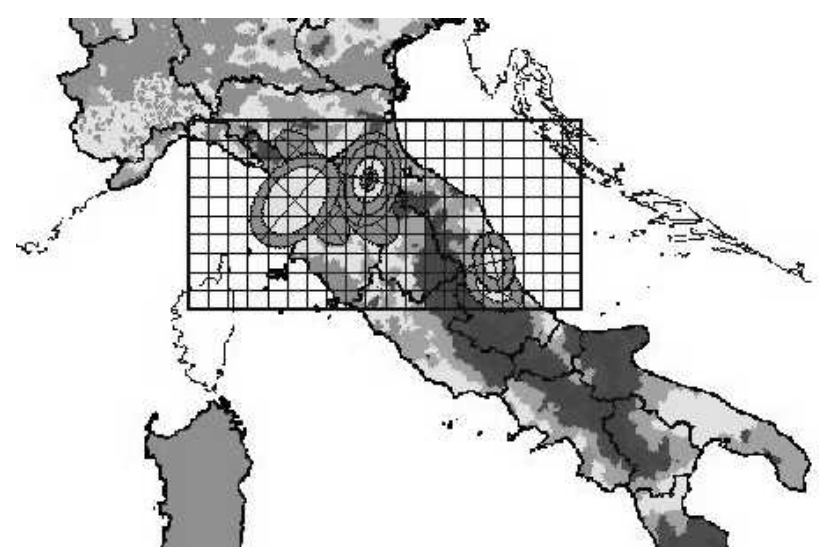

Figure 22.1. Earthquake generator.

In municipalities affected at time $t$ the vulnerability relations between accelerations and losses [36] according to the type (masonry or reinforced concrete), age, and maintenance of the buildings are used to estimate the number of cubic meters of destroyed properties. The economic loss of destroyed cubic meters of a building is defined as the cost for their reconstruction. Then it is possible to be independent of contingent pricing by considering the cost of reconstruction per cubic meters to be the monetary unit. In this way the simulation of time histories for possible earthquakes in the region produces the sets of economical losses and enables the design of an insurance program. It also enables us to determine in which way preventive retrofitting could decrease the losses: this is easily done by a consequent decrease of the vulnerability indices in the loss model. In this way it is possible to study the interplay between structural measures and risk sharing for an integrated risk management approach and to design an insurance system linked to incentives for retrofitting of the built environment. Our analysis was primarily concerned with the following. In its early version the Italian Design of Law 2793 (1998), to reduce the impact of natural disasters on the governmental budget, included in provisions for an insurance program against all natural hazards. It was intended not to make this insurance mandatory, but to make mandatory the extension of a fire insurance policy to all natural hazards, in a way similar to the French system (see section 22.3). In addition to tax incentives for such an insurance, it stipulated a maximum exclusion layer of $25 \%$, the creation of a pool of insurance companies with an appropriate reserve fund, e.g., corresponding to the annual average government payment for compensating losses (with some forms of state guarantee to be specified further), and linking of the premium to the premium for fire policy. This article was withdrawn, and later proposals are still the subject of discussion.

Starting from these principles, the case study intends to demonstrate how the model analyzes and offers the decision makers different policy options. Let us assume that an insurance company (this might be a pool of companies or the government itself acting as an insurer) covers a fraction, e.g., $q=0.75$, of earthquake losses. The rest $v=1-q$, according to the Italian Design of Law, would be compensated by the state. The state would also be expected to feed the reserve funds in case of excessive losses. 
The company has an initial catastrophe fund or a risk reserve $R^{0}$, which in general is characterized by a random variable dependent on past catastrophic events. It is also possible to analyze needs for the future as $R^{0}$ a policy variable. For example, taking $R^{0}=0$ enables us to evaluate the capacity of the region to accumulate risk reserves in the future. Assume that the time span consists of $t=\overline{1, T}, T=50$, time intervals. The stopping time $\tau$ is the time of the first catastrophe in the region within the time horizon $T$. The risk reserve (wealth) $R^{t}$ of the pool at time $t=\overline{1, T}$ is calculated according to (22.4):

$$
R^{t}=R^{t-1}+\sum_{j=1}^{m} \pi_{j}-\sum_{j=1}^{m} L_{j}^{t}\left(\omega_{t}\right) q
$$

where $q$ defines the coverage of the pool in affected municipalities $j$ at time $t, \pi_{j}$ is the premium rate from the municipality $j$, and $L_{j}^{t}\left(\omega_{t}\right)$ is the loss (damage) at $j$ caused by the simulated catastrophic event $\omega_{t}$ at time $t$. The value $L_{j}^{t}\left(\omega_{t}\right)$ depends on the event $\omega_{t}$, the content of $j$, mitigation measures, and deterioration of the built environment. The analytical structure of the probability distribution of the random variable $R^{t}$ is intractable; therefore, the methodology relies on Monte Carlo simulation.

Standard actuarial approaches calculate premiums in accordance with loss expectations. Therefore this study analyzed two policy options based on similar principles:

1. premiums based on the average damage over all municipalities (solidarity principle, bringing less exposed locations to pay premiums equal to more severely exposed ones, as in the spirit of the proposed insurance program); and

2. location-specific premiums based on average damage in the particular municipality, i.e., risk-based premiums.

However, the use of average losses may be misleading in the case of heavy-tailed distributions which are typical for catastrophic losses. The stochastic optimizations allows the analysis of different criteria and takes into account dependencies among location-specific losses. As an important example, a third policy option has been considered:

3. premiums calculated in a way that equalizes in a fair manner the risk of instability for the insurance company and the risk of premium overpayment for exposed municipalities. Besides this, it was important to analyze location-specific coverages and the amount of governmental compensation as a decision variable.

For option 3 it was assumed that the pool maximizes its wealth (risk reserves) taking into account the risks of the insolvency under the constraint on "fair" premiums. Fair premiums are defined according to the specified probability (say, once in every 100 years) of cases when paid premiums exceed actual claim sizes.

Accordingly, the goal function (22.6) for the pool at $t=\tau, R^{0}=0$, is defined as

$$
f^{\tau}(x, V, \omega)=V+\gamma \min \left\{0, R^{\tau}(x, \omega)-V\right\}+\delta \min \left\{0, R^{\tau}(x, \omega)\right\} .
$$

The stability of the welfare growth of municipalities can be written in the form of the chance constraints on overpayments

$$
P\left\{(1-q) L_{j}^{t}+L_{j}^{t} q_{j}<\pi_{j}\right\} \leq p, \quad \sum_{j=1}^{m} q_{j}=q,
$$




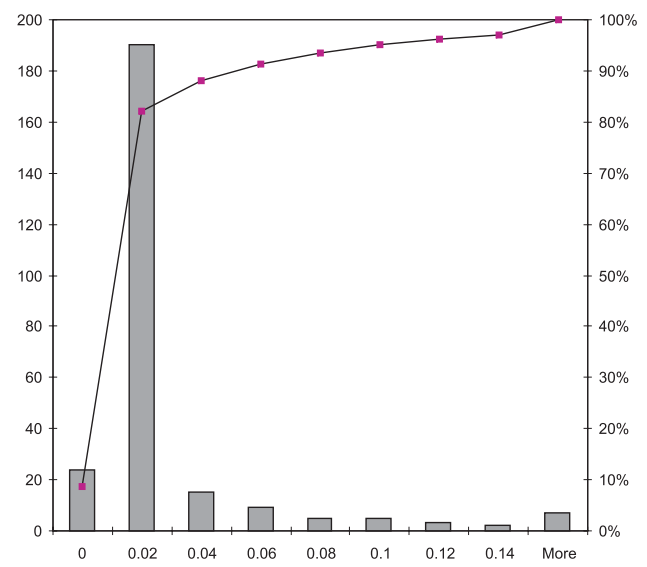

Figure 22.2. Distribution of municipality-specific premiums (per building volume/ municipality, percent terms).

where $x=\left(\pi_{1}, \ldots, \pi_{n}, q_{1}, \ldots, q_{n}\right), x \geq 0, p$ is a given "safety" level. The difference $q-q_{j}$ defines the partial coverages of some municipalities, which generates the demand for further increase of the compensation by the government. The wealth of municipalities at $t=\tau$ changes due to the insurance program from $W_{j}^{\tau}-L_{j}^{\tau}$ to $W_{j}^{\tau+}=W_{j}^{\tau}-L_{j}^{\tau}+\left(1-q+q_{j}\right) L_{j}^{\tau}-\pi_{j}$. The stochastic goal function (22.6) for municipality $j$ at $t=\tau$ is

$$
f_{j}^{\tau}\left(x, V_{j}, \omega\right)=V_{j}+\gamma_{j} \min \left\{0, W_{j}^{\tau+}-V_{j}\right\}+\delta_{j} \min \left\{0,\left(1-q+q_{j}\right) L_{j}^{\tau}-\pi_{j}\right\} .
$$

Figures 22.2-22.6 illustrate some numerical results. The number of simulations is shown on the vertical axis.

For option 1, where the burden of losses is equally distributed over the population, the simulation of catastrophic losses showed that the annual premium is equal to the flat rate of 0.02 monetary units (m.u.) per cubic meter of building.

For option 2, Figure 22.2 shows the distribution of municipality-specific premiums based on average damage in each municipality (or according to the municipality-specific risk). There is a prevailing number of municipalities (about 220) that have to pay $0.02-0.03$ m.u., which is close to the flat rate of 0.02 , as in option 1 . About 20 municipalities are at no risk at all ( 0 rate). Municipalities more exposed to the risk have to pay 0.04 and higher rates (more than 50 municipalities).

Figure 22.3 shows the distribution of the insurers' reserve (cumulated at $\tau$ within 50 years) at premiums of option 2. The volume of capital is shown on the horizontal axis. The probability of insolvency (when the risk reserve accumulated up to the catastrophe is not enough to compensate incurred losses) is indicated on the right-hand ordinate axis. There is a rather high probability of "small" insolvency (values $-90,-40$ occurred 190 and 90 times out of 500 fast simulations, as discussed in sections 22.2 and 22.7). High solvency (more than 500 m.u.) occurred in about $10 \%$ of the simulations. The size of insolvency would represent the cost to the government to cover losses uncovered by the pool. Another option may be to transfer a fraction of losses to international financial markets, as analyzed in [17]. 


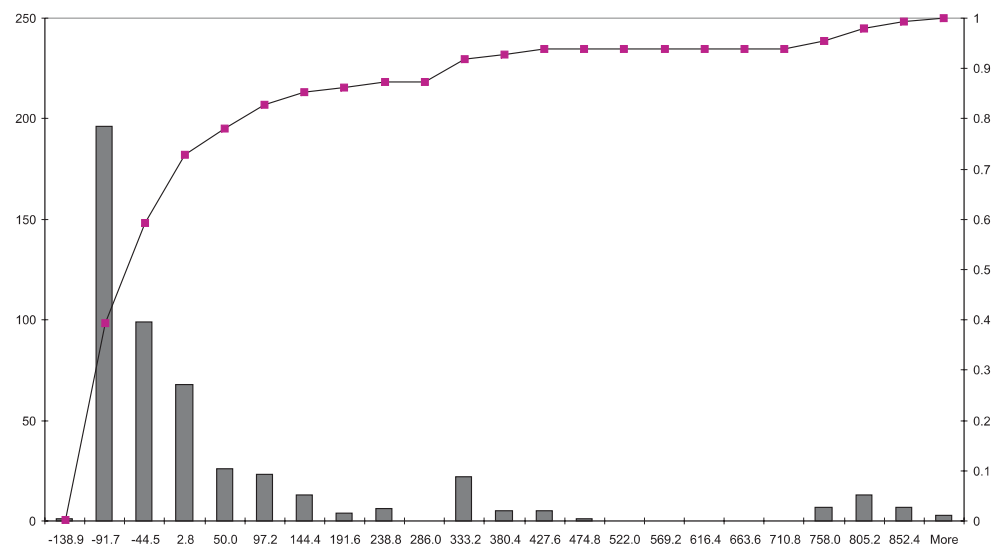

Figure 22.3. Distribution of insurer's reserve, options 1 and 2 (thousands m.u., 50 years).

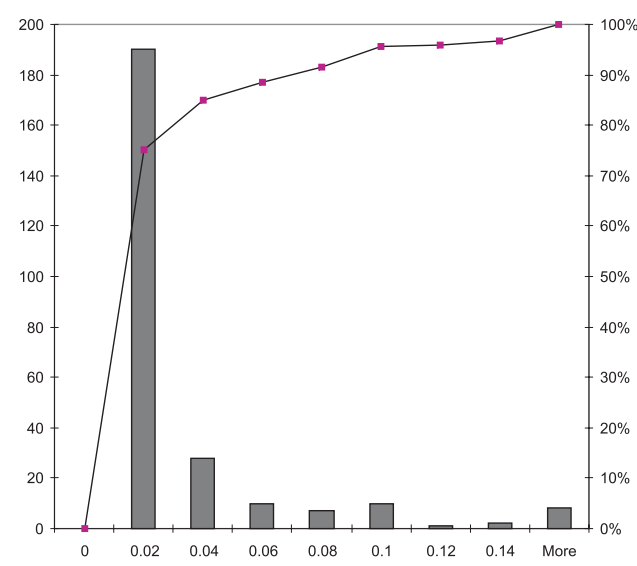

Figure 22.4. Distribution of fair premiums, option 3 (per building volume/ municipality, percent terms).

Figure 22.4 shows the distribution of premiums for option 3. According to this principle, most of the municipalities (190) have to pay close to the flat rate of 0.02-0.03 m.u. per cubic meter of a building. Rates of 0.04 and higher have to be paid by about 100 municipalities. In this case the highest premium rate is 0.5 , which is much lower than the highest rate of 1.2 of option 2. The distribution of the insurer's reserve in Figure 22.5 indicates also the improvement of the insurer's stability: the frequency of insolvency is considerably reduced.

Figure 22.6 is very illustrative. For each municipality it shows the optional premiums to be paid: the flat premium rate of 0.02 , the option 2 municipality-specific rate, and the fair premium of option 3. Many municipalities in all three options have to pay the premium rate, which is about the flat rate $(0.015-0.03)$. For quite a number of municipalities in option 2, the rate significantly exceeds the flat rate. For these municipalities special attention should 


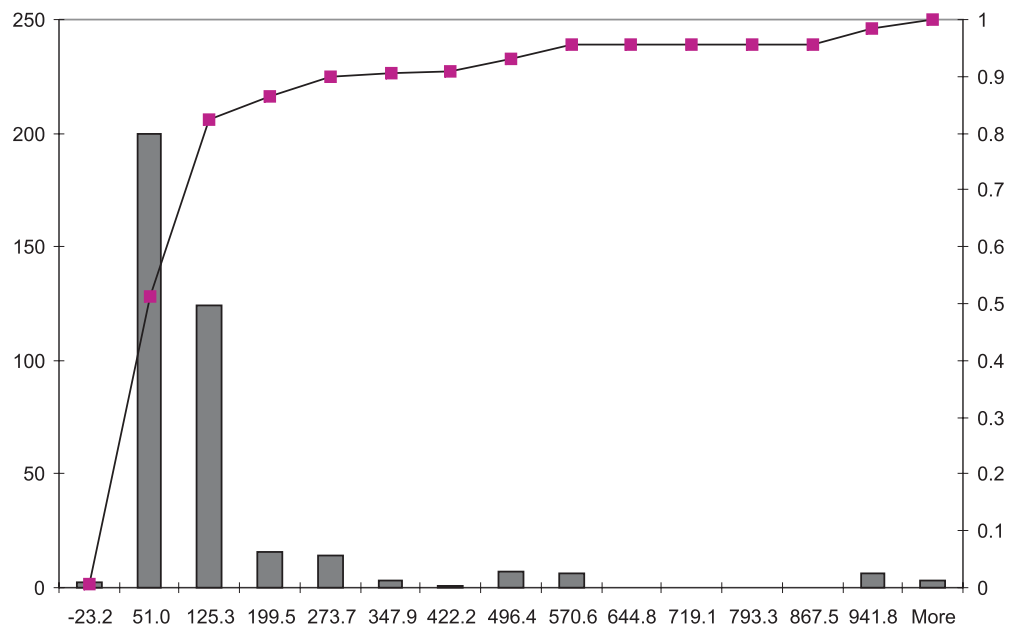

Figure 22.5. Distribution of insurers'reserve, option 3 (thousands m.u., over 50 years).

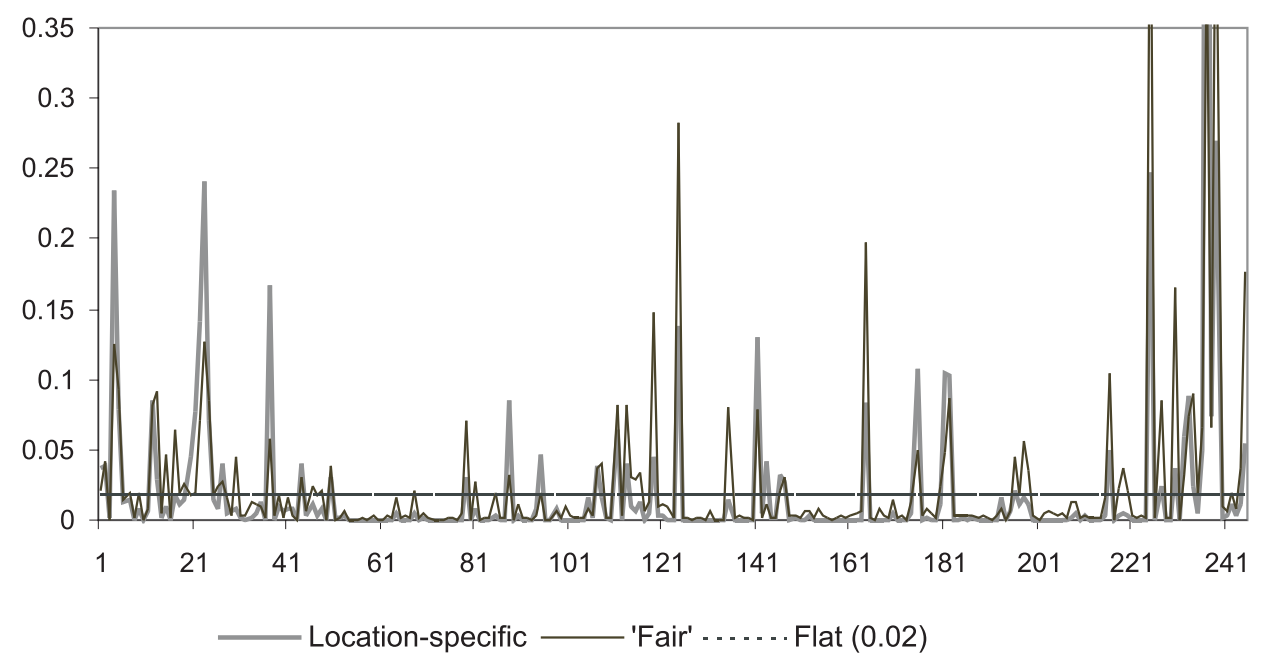

Figure 22.6. Comparison of options: municipality-specific, fair, and flat (0.02) premiums.

be given as to whether they are able to pay such high premiums. Option 3 allows one to take such individual constraints on overpayments into account and work out the efficient premiums both for insurer and municipalities.

\subsection{The solution procedure}

From the discussion in section 22.4, it follows that the welfare function $W(x, V)$ for the case study in the Tuscany region is a concave function assuming that $\tau$ and $L_{j}^{\tau}$ do not depend 
on $x$. In this case the minimization of $W(x, V)$ can be approximately solved by linear programming methods (see general discussion in [15]). The resulting linear approximation may prove to have extremely large dimensions due to the large number of scenarios for estimating the function being optimized. The main challenge arises in the case when $\tau$ and $L_{j}^{\tau}$ are implicit functions of $x$. Then we can only use the stochastic quasi-gradient (SQG) methods (see $[12,6])$. Let us outline only the main idea of these techniques. More details and further references are in $[14,15]$.

Assume that vector $x$ incorporates not only risk management decision variables $x$ but also $V$ and decisions affecting the efficiency of the sampling itself (for more detail, see $[16,39]$ ). An adaptive Monte Carlo optimization procedure (SQG method) searching for a solution minimizing $W(x)$ starts at any reasonable guess $x^{0}$. It updates the solution sequentially at steps $k=0,1, \ldots$, by the rule $x^{k+1}=x^{k}-\rho_{k} \xi^{k}$, where numbers $\rho_{k}>$ 0 are predetermined step sizes satisfying the condition $\sum_{k=0}^{\infty} \rho_{k}<\infty, \sum_{k=0}^{\infty} \rho_{k}^{2}=\infty$. For example, the specification $\rho_{k}=1 /(k+1)$ would formally suit. Random vector $\xi^{k}$ is an estimate of the gradient $W_{x}(x)$ or its analogs for nonsmooth function $W(x)$. This vector is easily computed from random observations of $W(x)$. For example, let $W^{k}$ be a random observation of $W(x)$ at $x=x^{k}$ and $\widetilde{W}^{k}$ be a random observation of $W(x)$ at $x=x^{k}+\delta_{k} h^{k}$. The numbers $\delta_{k}$ are positive, $\delta_{k} \rightarrow 0, k \rightarrow \infty$, and $h^{k}$ is an independent observation of the vector $h$ with components independent and uniformly distributed on $[-1,1]$. Then $\xi^{k}$ can be chosen as $\xi^{k}=\left[\left(\widetilde{W}^{k}-W^{k}\right) / \delta_{k}\right] h^{k}$. There is significant flexibility in choosing $\xi^{k}$ for estimating the gradient of $W(x)$ at $x=x^{k}$. Some of them may lead to fast convergence; others produce slow oscillating behavior. For example, the straightforward estimation of function $\Psi(x)$ in section 22.2 is time consuming. But due to formula (22.1) we can use the following procedure. Consider any sequence of numbers $\mu_{t}>0, t \geq 1$, $\sum_{t=1}^{\infty} \mu_{t}=1$. Step $k+1$ : choose $t_{k}$ with a probability $\mu_{t}$ from set $t \in\{1,2, \ldots\}$; generate $p_{k} \in[p, \bar{p}]$ and simulate claim $B_{k}^{t_{k}}$ by a catastrophe model. Calculate $\xi^{k}=\mu_{t_{k}}^{-1}[p(1-$ $\left.p)^{t_{k}-1} V_{t_{k}}^{\prime}\left(\min \left\{x^{k}, B_{k}^{t_{k}}\right\}-\pi t^{k}\right)\right] \eta^{k}$, where $V_{t}^{\prime}$ denotes the derivative of $V_{t}(\cdot)$, and $\eta^{k}=1$ if $x^{k} \leq B_{k}^{t_{k}}$, and $\eta^{k}=0$ otherwise. It is easy to see, e.g., from the discussion of the stochastic minimax problems in [12, p. 165], that $\mu_{t_{k}}^{-1}\left[p(1-p)^{t_{k}-1} V_{t_{k}}^{\prime}\left(\min \left\{x^{k}, B_{k}^{t_{k}}\right\}-\pi t^{k}\right)\right] \eta^{k}$ is an estimate of $\Psi^{\prime}\left(x^{k}\right)$; i.e., its expected value is $\Psi^{\prime}\left(x^{k}\right)$. The rate of asymptotic convergence of this method (when the number of observations $k \rightarrow \infty$ ) is similar to other sampling based procedures.

\section{Bibliography}

[1] A. Amendola, Y. Ermoliev, and T. Ermolieva, Earthquake risk management: A case study for an Italian region, in Proceedings of the Second EuroConference on Global Change and Catastrophe Risk Management: Earthquake Risks in Europe, J. Linnerooth-Bayer and A. Amendola, eds., International Institute for Applied Systems Analysis, Laxenburg, Austria, 2000.

[2] A. Amendola, Y. Ermoliev, T. Ermolieva, V. Gitits, G. Koff, and J. LinneroothBAYER, A system approach to modeling catstrophic risks and insurability, Natural Hazards J., 21 (2000), pp. 381-393. 
[3] K. Arrow, The theory of risk-bearing: Small and great risks, J. Risk Uncertainty, 12 (1996), pp. 103-111.

[4] P. Artzner, F. Delbaen, J.-M. Eber, and D. Heath, Coherent measures of risk, Math. Finance, 9 (1999), pp. 203-228.

[5] S. Baranov, B. Digas, T. Ermolieva, And V. Rozenberg, Earthquake Risk Management: Scenario Generator, Interim Report 02-025, International Institute for Applied Systems Analysis, Laxenburg, Austria, 2002.

[6] J. Birge And F. Louveaux, Introduction to Stochastic Programming, Springer Series in Operation Research, Springer-Verlag, New York, 1997.

[7] K. Borch, Equilibrium in a reinsurance market, Econ. J., 30 (1962), pp. 424-444.

[8] J. Cummins and N. Doherty, Can Insurer Pay for the "Big One"?, Working Paper, Wharton Risk Management and Decision Processes Center, University of Pennsylvania, Philadelphia, 1996.

[9] G. Dantzig, The Role of Models in Determining Policy for Transition to a More Resilient Technological Society, IIASA Distiguished Lecture Series 1, International Institute for Applied Systems Analysis, Laxenburg, Austria, 1979.

[10] C. Daykin, T. Pentikainen, and M. Pesonen, Practical Risk Theory for Actuaries, Monogr. Statist. Appl. Probab. 53, Chapman and Hall, London, 1993.

[11] P. Embrechts, C. Klueppelberg, AND T. Mikosch, Modeling Extremal Events for Insurance and Finance: Applications of Mathematics, Stochastic Modeling and Applied Probability, Springer-Verlag, Heidelberg, 2000.

[12] Y. ERmoliev and R. Wets, EDS., Numerical Techniques of Stochastic Optimization: Computational Mathematics, Springer-Verlag, Berlin, 1988.

[13] Y. Ermoliev, T. Ermolieva, G. MacDonald, and V. Norkin, Insurability of catastrophic risks: The stochastic optimization model, Optim. J., 47 (2000), pp. 251-265.

[14] Y. Ermoliev, T. Ermolieva, G. MacDonald, and V. Norkin, Stochastic optimization of insurance portfolios for managing exposure to catastrophic risks, Ann. Oper. Res., 99 (2000), pp. 207-225.

[15] Y. Ermoliev, T. Ermolieva, G. MacDonald, and V. Norkin, Problems on insurance of catastrophic risks, Cybernet. Syst. Anal., 2 (2001), pp. 220-234.

[16] T. ERmolieva, The Design of Optimal Insurance Decisions in the Presence of Catastrophic Risks, Interim Report 97-028, International Institute for Applied Systems Analysis, Laxenburg, Austria, 1997.

[17] T. Ermolieva, Y. Ermoliev, J. Linnerooth-Bayer, and I. Galambos, The role of financial instruments in integrated catastrophic flood management, in Conference Proceedings of the Eighth Annual Conference of the Multinational Finance Society, Garda, Italy, P. Theodossiou, ed., School of Business, Rutgers University, Camden, NJ, 2001. 
[18] T. Ermolieva, Y. Ermoliev, AND V. Norkin, Spatial Stochastic Model for Optimization Capacity of Insurance Networks under Dependent Catastrophic Risks: Numerical Experiments, Interim Report 97-028, International Institute for Applied Systems Analysis, Laxenburg, Austria, 1997.

[19] K. Froot, The Limited Financing of Catastrophe Risk: An Overview, Harvard Business School, Cambridge, MA, 1997.

[20] O. Giarini and H. Louberg, The Diminishing Returns of Technology, Pergamon Press, Oxford, UK, 1978.

[21] C. Gilber And C. Gouy, Flood management in France, in Flood Response and Crisis Management in Western Europe: A Comparative Analysis, U. Rosenthal and P. Hart, eds., Springer-Verlag, Berlin, 1998.

[22] V. Ginsburg AND M. Keyzer, The Structure of Applied General Equilibrium Models, MIT Press, Cambridge, MA, 1997.

[23] J. Grandell, Aspects of Risk Theory, Springer Series in Statistics: Probability and Its Applications, Springer-Verlag, Berlin, 1991.

[24] IIASA, Proposal for the Project on Flood Risk Management Policy in the Upper Tisza Basin: A System Analytical Approach, International Institute for Applied Systems Analysis, Laxenburg, Austria, 2000.

[25] The Impact of Catastrophes on Property Insurance, Insurance Service Office, New York, 1994.

[26] N. Jobst AND S. Zenios, The tail that wags the dog: Integrating credit risk in asset portfolios, J. Risk Finance, 3 (2001), pp. 31-43.

[27] N. Jobst and S. Zenios, The Tail That Wags the Dog: Integrating Credit Risk in Asset Portfolios, Working Paper 01-24, Wharton Financial Institutions Center, University of Pennsylvania, Philadelpha, 2001.

[28] P. Kleindorfer And H. Kunreuther, The Complementary Roles of Mitigation and Insurance in Managing Catastrophic Risks, Working Paper 98-14, Wharton School, Center for Financial Institutions, University of Pennsylvania, Philadelphia, 1997.

[29] H. Konno and H. YamaZaKi, Mean absolute deviation portfolio optimization model and its application to Tokyo stock market, Management Sci., 37 (1991), pp. 519-531.

[30] H. Kunreuther And R. Roth, Paying the Price: The Status and Role of Insurance against Natural Disasters in the United States, Joseph Henry Press, Washington, DC, 1998.

[31] J. Linnerooth-BAyer And A. Amendola, Global change, catastrophic risk and loss spreading, Geneva Papers Risk Insurance, 25 (2000), pp. 203-219. 
[32] L. MacKellar and T. Ermolieva, The IIASA Social Security Project Multiregional Economic-Demographic Growth Model: Policy Background and Algebraic Structure, Interim Report 99-007, International Institute for Applied Systems Analysis, Laxenburg, Austria, 1997.

[33] H. Markowitz, Mean Variance Analysis in Portfolio Choice and Capital Markets, Oxford, UK, Blackwell, 1987.

[34] Climate Change and Increase in Loss Trend Persistence, Technical Report, Munich RE, Munich, Germany, 1999.

[35] National Research Council, National Disaster Losses: A Framework for Assessment, National Academy Press, Washington, DC, 1999.

[36] V. Petrini, Pericolosita' sismica e prime valutazioni di rischio in Toscana, Report, CNR/IRRS, Milan, Italy, 1995.

[37] J. Pollner, Catastrophe Risk Management: Using Alternative Risk Financing and Insurance Pooling Mechanisms, Finance, Private Sector and Infrastructure Sector Unit, Working Paper 2560, Caribbean Country Department, Latin America and the Caribbean Region, World Bank, Washington, DC, 2000.

[38] A. Prekopa, Stochastic Programming, Kluwer Academic Publishers, Dordrecht, The Netherlands, 1995.

[39] E. L. Pugh, A gradient technique of adaptive Monte Carlo, SIAM Rev., 8 (1966), pp. 346-355.

[40] T. Rockafellar and S. Uryasev, Optimization of conditional value-at-risk, J. Risk, 2 (2000), pp. 21-41.

[41] J. M. Stone, A theory of capacity and the insurance of catastrophe risks, J. Risk Insurance, 40 (1973), pp. 231-244, 339-355.

[42] V. Rozenberg, T. Ermolieva, And M. Blizorukova, Modeling Earthquakes via Computer Programs, Interim Report 01-068, International Institute for Applied Systems Analysis, Laxenburg, Austria, 2001.

[43] G. Walker, Current developments in catastrophe modelling, in Financial Risks Management for Natural Catastrophes, N. Britton and J. Oliver, eds., Griffith University, Brisbane, Australia, 1997. 


\section{Additional copies}

Further copies of this IIASA Reprint are available online at www.iiasa.ac.at/Publications

Hard copies are also available. Orders must include the publication number and should be sent to the Publications Department, International Institute for Applied Systems Analysis, A-2361 Laxenburg, Austria.

Telephone: +432236807

Telefax: +432236 71313

E-mail: publications@iiasa.ac.at 
International Institute for Applied Systems Analysis

Schlossplatz 1, A-2361 Laxenburg, Austria

Tel: +432236807 Fax: +43223671313

I I AS A www.iiasa.ac.at 